\title{
Nivel de comprensión del Sistema de Perfilado Nutricional NutriScore para las elecciones alimentarias y su relación con el nivel educativo
}

\section{Level of understanding of the NutriScore Nutrient Profiling System for food choices and its association with educational level}

\author{
Natalia Belén Bertorello ${ }^{1}$ (D) , Constanza Rodríguez Junyent ${ }^{1}$, Valentina Barbero ${ }^{1}$, Antonella Ninin ${ }^{1}$ \\ 1 Universidad Católica de Córdoba, Facultad de Ciencias de la Salud, Licenciatura en Nutrición. \\ Correspondencia: Lic. Natalia Belén Bertorello. E-mail: licnataliabertorello@gmail.com
}

\section{Resumen}

INTRODUCCIÓN: El Sistema de Perfilado Nutricional (SPN) NutriScore a través de su etiquetado frontal permite a los consumidores realizar un juicio rápido de la calidad nutricional de los alimentos, favoreciendo elecciones alimentarias más saludables.

OBJETIVO: Determinar el nivel de comprensión del SPN NutriScore para las elecciones alimentarias y su asociación con el nivel educativo, de los referentes beneficiarios de la Fundación Banco de Alimentos de Córdoba (FBAC), en la Ciudad de Córdoba durante 2020.

MATERIALES Y METODOS: Estudio observacional, analítico, de corte transversal. Participaron 45 referentes beneficiarios de FBAC. Se utilizó una encuesta validada, estructurada y administrada por entrevistador. El instrumento fue adaptado a los patrones alimentarios de la población Argentina, seleccionando 3 categorías de alimentos comúnmente consumidos y de diferentes calidades nutricionales. El análisis de los datos se realizó con Stata v14. Para las variables categóricas se utilizaron frecuencias relativas y absolutas; mientras que para variables continuas se usaron medidas de posición central y dispersión. Por último, se aplicó el Test de Fisher para evaluar asociación entre las variables $(\mathrm{p}<0,05)$.

RESULTADOS: El $78 \%$ de los encuestados fueron mujeres, con un promedio de 47 años. Se pudo destacar que $71 \%$ de los encuestados presentó un nivel medio-alto de comprensión del SPN. El nivel educativo no se asoció al nivel de comprensión de NutriScore.

CONCLUSIÓN: SPN NutriScore es comprendido por una parte importante de los consumidores, por lo que podría implementarse como una herramienta nutricional, con la finalidad de prevenir enfermedades y promover la salud.

Palabras claves: NutriScore; Sistema de Perfilado Nutricional, Nivel de comprensión, Nivel educativo, Córdoba.

\begin{abstract}
INTRODUCTION The NutriScore Nutrient Profiling System (NPS) through its front labeling allows consumers to make a quick judgment of the nutritional quality of food, favoring the healthiest food choices. OBJECTIVE: To determine the level of understanding of the NPS NutriScore for food choices and its association with educational level, of the beneficiaries referent of the "Fundación Banco de Alimentos de Córdoba" (FBAC), in the City of Córdoba during 2020.
\end{abstract}


MATERIALS AND METHODS: Observational, analytic and cross-sectional study. 45 beneficiaries referent of the FBAC participated. A validated, structured and interviewer-administered survey was used. The instrument was adapted based on the food patterns of the Argentine population, selecting 3 categories of commonly consumed foods, considering the availability of products in the market and of different nutritional qualities. The data analysis was carried out with Stata v14. For categorical variables relative and absolute frequencies were used; while for continuous variables measures of central position and dispersion were used. Finally, the Fisher test was applied to evaluate the association between the variables ( $\mathrm{p}<0.05)$. RESULTS: $78 \%$ of those surveyed were women, with an average of 47 years. It was noted that $71 \%$ of the respondents presented a médium-high level of understanding of the NPS. Level educational was not associated with NutriScore level of understanding.

CONCLUSION: The NPS NutriScore is understood by an important part of consumers, so it could be implemented as a nutritional tool, in order to prevent diseases and promote health.

KeyWords: NutriScore; Nutrient Proling System, Level of understanding, Educational level, Córdoba.

\section{Introducción}

Frente a la problemática mundial de sobrepeso, obesidad y otras enfermedades crónicas no transmisibles, la Organización Mundial de la Salud (OMS) propone para su prevención y control entre otras medidas la implementación eficiente del etiquetado nutricional $(\mathrm{EN})^{1}$.

Las elecciones de alimentos saludables se ven afectadas por los escasos conocimientos nutricionales de la población, la falta de tiempo en el momento de la compra y la complejidad en la forma de presentar la información nutricional, dando lugar al EN como elemento primordial a la hora de elegir un alimento ${ }^{2}$.

La evidencia demuestra que en general la presencia del EN frontal resulta más eficaz que la etiqueta tradicional para ayudar a los consumidores a seleccionar aquellos alimentos que califican como más saludables según el sistema de perfil de nutrientes (SPN). El etiquetado frontal, persigue como objetivo, reducir los esfuerzos cognitivos y el tiempo para procesar la información de las etiquetas, facilitando la elección de alimentos más saludables y de esta forma, contribuir a la prevención del sobrepeso, obesidad y otras enfermedades vinculadas a una alimentación inadecuada ${ }^{3}$.

En Latinoamérica, numeros países ya han sancionado normativa de EN frontal de cáracter obligatoria. Ecuador fue el pionero en la implementación del sistema semáforo, Chile implementó el símbolo octogonal con la descripción "Alto en" para calificar a sus alimentos y México implementó el "sello nutrimental"4. Si bien Argentina aún no cuenta con SPN, actualmente se está evaluando en la cámara de diputados la Ley del Etiquetado frontal de alimentos con la implementación de sellos negros de advertencia de los niveles críticos de sodio, grasas trans, azúcares o calorías ${ }^{5}$.

Por otra parte, Francia y Bélgica decidieron establecer un EN frontal de tipo resumen de 5 colores "NutriScore" (llamado también 5C). El logotipo cromático y alfabético de NutriScore permite informar a los consumidores de manera simple sobre la calidad nutricional de los alimentos e incitar a la industria alimentaria a mejorar la composición nutricional de los alimentos que producen a través de las reformulaciones e innovaciones para obtener una mejor puntuación en la escala de colores del NutriScore ${ }^{6}$.

Las elecciones alimentarias al momento de comprar afectan la salud de toda la sociedad. En muchos casos el consumidor final es quien elige su alimento y tiene la capacidad de cambiar esa conducta de acuerdo al etiquetado. En otras situaciones y en contextos de vulnerabilidad económica, las familias no tienen el poder de decidir sobre su alimentación, dejándolo a cargo de otros (estado $\mathrm{u}$ organizaciones no gubernamentales $)^{7}$.

En la Ciudad de Córdoba, el Banco de Alimentos de Córdoba (FBAC), una organización sin fines de lucro, se encarga de distribuir alimentos que han sido sacados de la circulación comercial entre comedores y organizaciones sociales, que colaboran en la alimentación de personas con inseguridad alimentaria ${ }^{8}$. De esta manera, los referentes de las organizaciones beneficiarias de la FBAC toman decisiones en el acceso alimentario de gran parte de niños y adultos que asisten a dichos comedores. Considerando que, en la actualidad, el sobrepeso y la obesidad, constituyen uno de los principales problemas de salud pública ${ }^{9}$, este trabajo pretende determinar el nivel de comprensión del SPN NutriScore para las elecciones alimentarias y su asociación con el nivel educativo, de los referentes beneficiarios de la FBAC, en la Ciudad de Córdoba durante 2020.

Revista Methodo: Investigación Aplicada a las Ciencias Biológicas. Universidad Católica de Córdoba. Jacinto Ríos 571 Bo Gral. Paz. X5004FXS. Córdoba. Argentina. Tel.: (54) 3514517299 / Correo: methodo@ucc.edu.ar / Web: methodo.ucc.edu.ar | ARTICULO ORIGINAL Rev. Methodo 2021;6(4):168-174. 


\section{Materiales y método}

La investigación se llevó a cabo con los referentes de comedores y merenderos beneficiarios de la FBAC, como parte del proyecto de investigación "Sistema de Perfilado Nutricional y su impacto como herramienta de Educación Alimentaria Nutricional".

Se realizó un estudio de tipo observacional, analítico y de corte transversal. El muestreo fue no probabilístico por conveniencia, la encuesta se envió a un total de 50 referentes beneficiarios del FBAC, pero sólo 45 referentes la respondieron. Como criterios de inclusión el referente debía tener acceso a la aplicación de WhatsApp.

Para la recolección de los datos se utilizó un instrumento ya validado por otras investigaciones llevadas a cabo en 12 países ${ }^{10}$, adaptándolo a nuestra población en estudio. Para la construcción del instrumento, se realizaron los siguientes procedimientos que se detallan a continuación. En primer lugar, se consideraron los patrones alimentarios actuales de la población Argentina, analizados por Zapata et al. ${ }^{11}$, que evidencian una disminución en el consumo aparente de frutas, vegetales, harina de trigo, legumbres, carne vacuna y leche; y aumento en el consumo de masas de tartas y empanadas, fideos, galletitas, yogur, carne porcina, productos cárnicos semielaborados, gaseosas, jugos y alimentos listos para consumir. De esta manera, se seleccionaron 3 categorías de alimentos comúnmente consumidos que fueron pastas secas, galletas dulces y yogures; se contempló que los productos estuvieran disponibles en el mercado y presentaran diferentes calidades nutricionales. Para la elección de los alimentos que formaron parte de la encuesta se analizaron sus macronutrientes (carbohidratos, grasas totales, saturadas, trans, colesterol y proteínas), además se tuvo en cuenta el contenido de sodio y fibra aportado por cada alimento. A partir de su calidad nutricional se dividieron en 3 grupos: calidad nutricional alta, media y baja. Es decir, se obtuvo un producto alimenticio según cada nivel de calidad nutricional y por cada categoría de alimentos mencionada anteriormente, en total se utilizaron 9 alimentos para formular la encuesta.

En segundo lugar, se modificó el diseño de los packaging de estos alimentos con el objetivo de no mostrar otra información relevante como lista de ingredientes, alegaciones nutricionales o marcas, que puedan influenciar en las respuestas de las encuestas. En otras palabras, se le borró la marca comercial y se le añadió la denominación de la categoría del producto y el logotipo NutriScore con la letra y color correspondiente a la calidad nutricional de cada alimento (Anexo 1).
De esta manera, se construyó una encuesta estructurada de carácter observacional apta para ser administrado por un entrevistador. Por cada producto alimentario, los participantes podían elegir entre las opciones de "alta calidad nutricional", "medio calidad nutricional y "baja calidad nutricional", también se incorporó la opción "No sé". Por motivos de público conocimiento de la pandemia mundial de COVID 19 transcurrida en el año 2020, la encuesta se realizó vía WhatsApp. Además, el instrumento permitió extraer datos biosocioculturales, como sexo, edad y nivel educativo.

Para la categorización de la variable comprensión de NutriScore, se establecieron tres categorías. El nivel alto de comprensión, se tomó cuando siete a nueve interpretaciones del etiquetado frontal de grupos de alimentos coincidieron con el significado del mensaje, el nivel medio de comprensión, cuando coincidieron cuatro a seis interpretaciones y el nivel bajo de comprensión, se consideró tres o menos interpretaciones acertadas del mensaje.

Para el análisis de los datos, se utilizó en una primera instancia la estadística descriptiva según la naturaleza de las variables. Para las variables categóricas se utilizó frecuencia relativa $\mathrm{y}$ frecuencia absoluta y para las variables cuantitativas se usó medidas de posición central y de dispersión como media y desvió estándar, respectivamente. En una segunda instancia, para analizar si el nivel de comprensión del SPN NutriScore estaba asociado al nivel educativo de los referentes se realizó el Test de Fisher. Los análisis estadísticos se realizaron con el programa estadístico Stata v14. Se trabajó con un nivel de significación de 0,05.

\section{Consideraciones éticas}

El protocolo de trabajo se basó en las pautas internacionales de la Declaración de Helsinki y la ley Nacional $N^{\circ} 26.529$ (Derechos del Paciente en su Relación con los Profesionales e Instituciones de la Salud). El presente trabajo forma parte del proyecto de investigación "Sistema de Perfilado Nutricional y su impacto como herramienta de Educación Alimentaria Nutricional", el cual cuenta con la aprobación del Comité Institucional de Ética de la Investigación en Salud (C.I.E.I.S). La participación de los sujetos del estudio fue voluntaria. 


\section{Resultados}

Los 45 participantes respondieron todas las preguntas de la encuesta. Las características individuales de la muestra estudiada se detallan en la (Tabla 1). La muestra quedo conformada por un $78 \%(n=35)$ de mujeres y el resto correspondieron al sexo masculino. La edad promedio de la población fue de 47 años, concentrándose el mayor grupo entre 51 a 60 años $(26.5 \%)$. En relación al nivel educativo, el $85 \%$ declaró tener un nivel de estudios entre secundario y superior. Mientras que sólo el $15 \%$ presentó un nivel educativo primario. En relación al nivel de comprensión del SPN NutriScore, se destaca que un $53 \%$ de los encuestados presentaron un nivel medio de comprensión, un $29 \%$ un nivel bajo de comprensión de NutriScore y un $18 \%$ un nivel alto de comprensión.

Tabla 1. Características biosociodemográficas de los referentes beneficiarios de la FBAC

\begin{tabular}{lcc}
\hline Características Generales & N & $\%$ \\
\hline Sexo & & \\
Femenino & 35 & 78 \\
Masculino & 10 & 22 \\
\hline Edad & & \\
$21-30$ años & 7 & 15.5 \\
$31-40$ años & 8 & 18 \\
$41-50$ años & 10 & 22 \\
$51-60$ años & 12 & 26.5 \\
$61-70$ años & 8 & 18 \\
\hline Nivel educativo & & \\
Primario & 7 & 15 \\
Secundario & 21 & 47 \\
Superior & 17 & 38 \\
\hline
\end{tabular}

Un $64 \%$ de los encuestados que analizaron los packaging, visualizó el logotipo NutriScore, mientras que menos de la mitad no lo logró o no está seguro de haberla visualizado.

Por último, se analizó el nivel de comprensión del SPN NutriScore según el nivel educativo de los participantes. De esta manera, se determinó que el $49 \%$ de los entrevistados pertenecientes a los niveles educativos secundario y superior, tuvieron un nivel de comprensión medio del NutriScore. A diferencia del nivel educativo primario en donde hubó mayor proporción de nivel de comprensión bajo (9\%). El mayor porcentaje de encuestados que tuvieron un nivel de comprensión alta, fueron aquellos con un nivel educativo superior (9\% en nivel superior, versus $2 \%$ en nivel educativo primario) (Figura 1). Sin embargo, luego de realizar el test de Fisher no se encontró una asociación significativa entre las variables $(\mathrm{p}=0,22)$.
Figura 1. Relación entre el nivel educativo de los encuestados y nivel de comprensión de NutriScore.

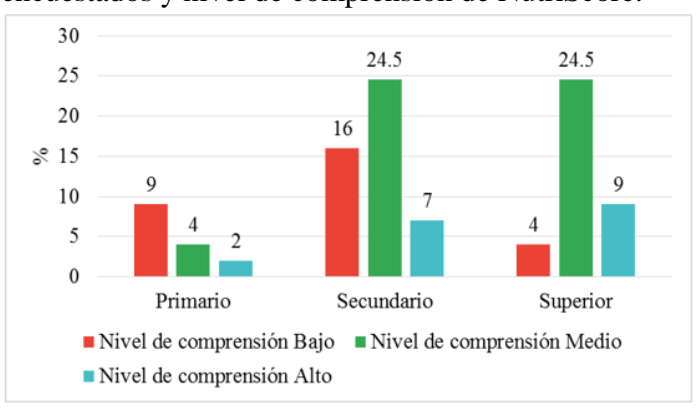

\section{Discusión}

En este estudio se puede destacar que un $71 \%$ de los encuestados presentó un nivel medio-alto de comprensión del SPN NutriScore. Estos resultados son coincidentes con un estudio realizado en Argentina en donde se demostró superioridad de interpretación del SPN NutriScore frente a otros etiquetados frontales ${ }^{12}$. Además, un estudio llevado a cabo en Francia arrojó que la etiqueta que contenía el SPN NutriScore, resultó la más fácil de identificar y la que requería la menor cantidad de esfuerzo y tiempo para comprender ${ }^{13}$.

Esta superioridad de comprensión del SPN, podría explicarse por dos motivos. En primer lugar, el sistema de etiquetado NutriScore es más comprensible de forma inmediata para los consumidores gracias a la indicación de los colores, lo que permitiría elegir alimentos con más claridad en la composición de kilocalorías, azúcar, grasas y sal en unas condiciones en las que el tiempo es limitado ${ }^{14}$. En estudios previos, se ha demostrado que el uso de colores es clave con respecto a los rasgos sobresalientes del EN frontal, ya que los colores tienden a captar la atención ${ }^{15-17}$. Además, el uso de la conocida escala policromática verde-roja podría ser una característica importante de la codificación de colores. Los colores verde y rojo, correspondientes a las señales reconocidas, pueden ser más fáciles de entender e interpretar, estando el verde asociado con la seguridad y una señal de "avance", y el rojo asociado con el peligro y la señal de "alto" ${ }^{18,19}$. Por lo tanto, la presencia de un SPN codificado por colores, como el NutriScore puede ser eficaz en diferentes etapas del procesamiento de la información: en una etapa temprana al llamar la atención sobre la etiqueta y en una etapa posterior educando para la comprensión ${ }^{20}$.

En segundo lugar, SPN NutriScore aporta información que resume la calidad nutricional global del producto y esto podría estar vinculado a 
una interpretación con menor trabajo cognitivo sobre la información nutricional aportada ${ }^{12}$.

Además, en nuestro estudio se observó que más de la mitad de la población encuestada visualizó el logotipo del SPN NutriScore al momento de la encuesta. No obstante, un estudio realizado en Países Bajos afirma que, aunque el EN en la parte frontal del envase ayude a los consumidores a realizar elecciones más saludables, la falta de atención a esas etiquetas puede limitar su eficacia ${ }^{21}$.

Retomando el estudio realizado en Argentina, el cual fue usado como referencia para la construcción del instrumento utilizado en este estudio, no se observaron diferencias significativas en la comprensión del SPN NutriScore con respecto al sexo, edad o nivel educativo ${ }^{12}$. Estos resultados son consistentes con nuestra investigación, ya que se demostró que el nivel de comprensión de NutriScore no varió en función del nivel educativo. De hecho, una de las fortalezas del SPN NutriScore, destacado en estudios previos, fue la efectividad que mostró este SPN para comparar favorablemente etiquetas en sujetos con niveles educativos más bajos, que han sido identificados por tener potencialmente más dificultades para entender las $\mathrm{EN}^{22}$.

Con respecto a las características biológicas de la población en estudio, participaron un $78 \%$ de mujeres y un $22 \%$ de hombres, a diferencia de otros estudios de referencia ${ }^{12,13}$ donde su distribución fue más homogénea. Probablemente esta diferencia hallada se deba al rol protagónico de la mujer como encargada del comedor comunitario ${ }^{23}$.

La fortaleza de nuestra investigación, se puede destacar que no se mostró otra información relevante, como lista de ingredientes, alegaciones nutricionales o marcas, que podrían haber tenido una influencia en las respuestas de las encuestas. Además, se utilizó como instrumento, una encuesta validada por otros trabajos de investigación y puede ser insumo para replicar la metodología.

El presente estudio tiene algunas limitaciones; la población estudiada, se limitó a los referentes de organizaciones beneficiarias de la FBAC que tenían celular y acceso a la aplicación WhatsApp, las conclusiones no pueden ser extrapoladas a la población en general, si no que caracterizan aquellas que lo hicieron. Además, debe agregarse el escaso tamaño muestral y que la encuesta no fue anónima.

Otras de las limitaciones que presenta el estudio, es que se valoró la interpretación de un grupo acotado de alimentos disponibles y no la interpretación al momento de compra, donde se presenta mayor variedad de productos; se trata de una situación experimental. Por lo tanto, los resultados no se pueden considerar un reflejo exacto de lo que sucedería en un contexto real.

Son necesarias más investigaciones, para evaluar el impacto de la utilización de la información nutricional en el SPN NutriScore sobre los hábitos de compra y consumo real.

\section{Objetivo}

Determinar el nivel de comprensión del SPN NutriScore para las elecciones alimentarias y su asociación con el nivel educativo, de los referentes beneficiarios de la Fundación Banco de Alimentos de Córdoba (FBAC), en la Ciudad de Córdoba durante 2020 .

\section{Conclusión}

La comprensión del SPN NutriScore como herramienta de educación nutricional estuvo reflejado en el $71 \%$ de los encuestados que presentó un nivel medio y alto de su comprensión, por otra parte, el nivel educativo no tuvo asociación con aquella cualidad. Lo que destacaría la superioridad de este SPN al brindar información de manera clara al momento de la elección alimentaria.

\section{Bibliografía}

1. World Health Organization. Follow-up to the Political Declaration of the High-level Meeting of the General Assembly on the Prevention and Control of Non communicable Diseases. World Health Assembly, 66, 2013. [Consulta: 26 de marzo de 2020]. Disponible en:https://apps.who.int/iris/handle/10665/150 161

2. León-Flándeza K, Prieto-Castillob L, RoyoBordonada M. Semáforo nutricional: conocimiento, percepción y utilización entre los consumidores de Madrid, España. Rev Esp Nutr Hum Diet. 2015; 19:97-104. [Consulta: 27 de marzo de 2020]. Disponible en: https://scielo.isciii.es/scielo.php?script=sci_a rttext\&pid=S2174-51452015000200006

3. Proyecto de Ley: Etiquetado frontal informativo de alimentos o sustancias aptas para consumo humano. Régimen. 2019. Diputados Argentinos. [Consulta: 28 de marzo de 2020]. Disponible en: https://www.diputados.gov.ar/proyectos/proy ectoTP.jsp?exp=4995-D-2019

4. Valverde-Aguilar M, Espadín-alemán CC, Torres-Ramos NE, Liria-Domínguez R. Preferencia de etiquetado nutricional frontal: 
octógono frente a semáforo GDA en mercados de Lima, Perú. Acta Med Peru. 2018; 35:145152

5. Senado Argentina. Julio Cobos: Argentina avanza hacia una ley de etiquetado de alimentos. Buenos Aires, 2020. [Consulta: 29 de octubre de 2020] Disponible en: www.senado.gob.ar/prensa/18890/noticias

6. Galán P, Babio N, Salas-Salvado J. NutriScore: el logotipo frontal de información nutricional útil para la salud pública de España que se apoya sobre bases científicas. Nutr Hosp. 2019; 36:1213-1222. [Consulta: 18 de mayo de 2021]. Disponible en: https://scielo.isciii.es/scielo.php?script=sci_a rttext\&pid=S0212-16112019000500030

7. Scribano A, Boito E. La ciudad sitiada: una reflexión sobre imágenes que expresan el carácter neo-colonial de la ciudad. Revista Actuel Marx Intervenciones. 2010;9.

8. Banco de Alimentos de Córdoba. Córdoba. [Consulta: 4 mayo de 2020]. Disponible en: https://www.bancodealimentoscba.org.ar/

9. Custodio J, Elizathe L, Murawski B, Rutsztein G. Obesidad en Argentina: un desafío pendiente. Políticas de salud pública y tasas de prevalencia. Rev. Mex. de trastor. aliment. 2015; 36:137-142.

10. Egnell M, Talati Z, Hercberg S, Pettigrew S, Julia C. Objective Understanding of Front-ofPackage Nutrition Labels: An International Comparative Experimental Study across 12 Countries. Nutrients. 2018; 10:1542.

11. Zapata ME, Rovirosa A, Carmuega E. Cambios en el patrón de consumo de alimentos y bebidas en Argentina, 1996-2013. Salud Colect. 2016; 12:473-486.

12. Galan P, Egnell M, Britos S, Borg A, Pettigrew S, Hercberg S. Evaluación de la comprensión objetiva de 5 modelos de etiquetado frontal de alimentos en consumidores argentinos: resultados de un estudio comparativo. Diaeta. 2019; 37:20-30.

13. Ducrot P, Mejean C, Julia C, Kesse-Guyot E, Touvier M, Fezeu L, Hercberg S, Péneau S. Effectiveness of Front-Of-Pack Nutrition Labels in French Adults: Results from the NutriNet- Sante Cohort Study. PlosOne. 2015; 10:e0140898.

14. Borgmeier I, Westenhoefer J. Impact of different food label formats on healthiness evaluation and food choice of consumers: a randomized-controlle study. BMC Public Health 2009; 9: 184.

15. Ducrot P, Julia C, Mejean P, Kesse-Guyot E, Touvier M, Fezeu LK, Hercberg S, Péneau S. Impact of Different Front-of-Pack Nutrition Label son Consumer Purchasing Intentions A Randomized Controlled Trial. Am J Prev Med. 2016; 50:627-636.

16. Hersey JC, Wohlgenant $\mathrm{KC}$, Arsenault JE, Kosa KM, Muth MK. Effects of front-ofpackage and shelf nutrition labeling systems on consumers. Nutr Rev. 2013; 71:1-14.

17. Antúnez L, Giménez A, Maiche A, Ares G. Influence of interpretation aids on attentional capture, visual processing, and understanding of front-of-package nutrition labels. J Nutr Educ Behav. 2015; 47:292-299.

18. Julia C, Peneau S, Buscail C, Gonzalez R, Touvier M, Hercberg S. Perception of different formats of front-of-pack nutrition labels according to sociodemographic, lifestyle and dietary factors in a French population: Cross-sectional study among the NutriNet-Sante cohort participants. BMJ Open. 2017; 7: e016108.

19. Vasiljevic M, Pechey R, Marteau TM. Making food labels social: The impact of colour of nutritional labels and injunctive norms on perceptions and choice of snack foods. Appetite. 2015; 91:56-63.

20. Becker MW, Bello NM, Sundar RP, Peltier C, Bix L. Front of pack labels enhance attention to nutrition information in novel and commercial brands. Food Policy. 2015; 56:7686.

21. van Herpen E, van Trijp HCM. Front-of-pack nutrition labels. Their effect on attention and choices when consumers have varying goals and time constraints. Appetite. 2011; 57:148160 .

22. Cowburn G, Stockley L. Consumer understanding and use of nutrition labelling: a systematic review. Public Health Nutr. 2005; 8:21-28.

23. Santarsiero LH. Comedores comunitarios en la ciudad de la Plata: organización social e intervención alimentaria estatal en el espacio barrial. Pilquen. 2013; 16:1-13.

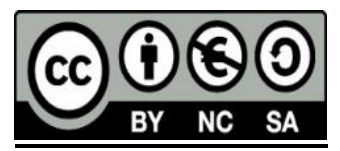


Bertorello N B, Rodríguez Junyent C, Barbero V, Ninin A. Nivel de comprensión del Sistema de Perfilado Nutricional NutriScore para las elecciones alimentarias y su relación con el nivel educativo.

Anexo 1. Construcción del Diseño del Packaging

\section{Pastas Secas}

Alta calidad nutricional

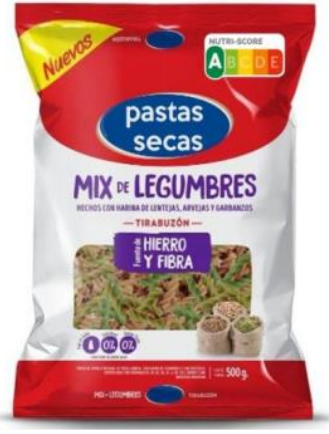

Galletas dulces

$\underline{\text { Alta calidad nutricional }}$

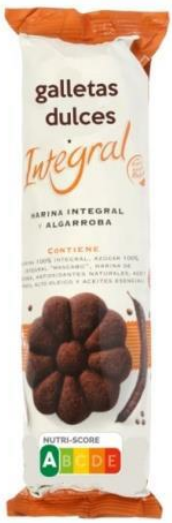

\section{Yogures}

$\underline{\text { Alta calidad nutricional }}$

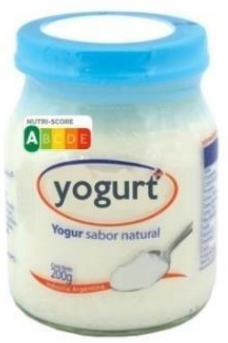

\section{$\underline{\text { Media calidad nutricional }}$}

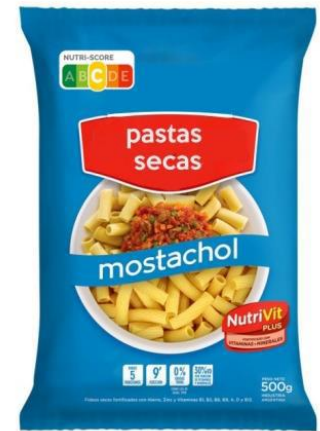

Baja calidad nutricional

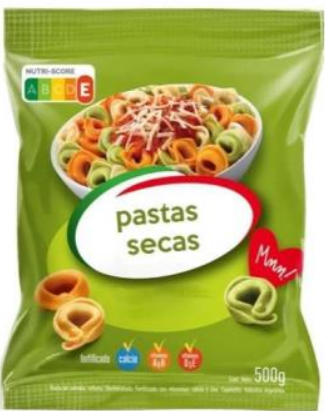

$\underline{\text { Media calidad nutricional }}$

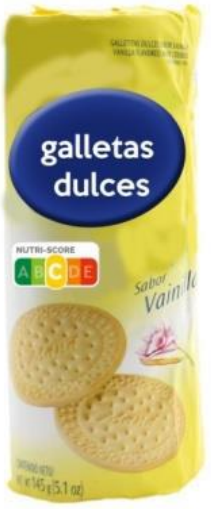

$\underline{\text { Baja calidad nutricional }}$

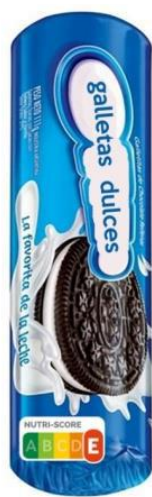

Media calidad nutricional

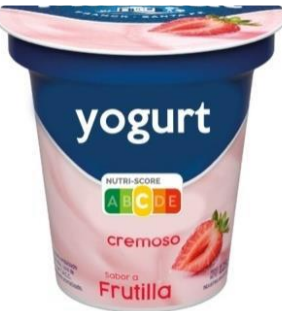

Baja calidad nutricional

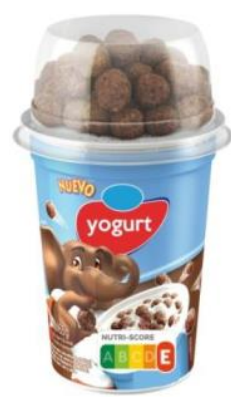

